Recepción: 20 / 04 / 2017

Aceptación: 20 / 05 / 2017

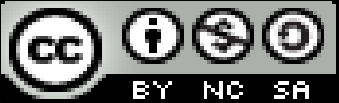

Publicación: 15 / 06 / 2017

\title{
Evaluación de la calidad físico-químico en agua y sedimentos del estero salado en el noroeste de América Latina
}

\author{
Evaluation of the physical-chemical quality in water and sediments of the salt \\ estuary in northwestern Latin America
} Avaliação de água físico-químicas e qualidade do sedimento no estuário salgado no
noroeste da América Latina

Magaly Peñafiel ${ }^{\mathrm{I}}$ magaly.penafielp@ug.edu.ec

Alida Vallejo ${ }^{\text {II }}$ alida.vallejol@ug.edu.ec

Judith Chalen III judith.2304@hotmail.com

Correspondencia: magaly.penafielp@ug.edu.ec

Docente de la Facultad de Ciencias Médicas, Universidad de Guayaquil, Guayaquil, Ecuador.

Docente de la Facultad de Ciencias Médicas, Universidad de Guayaquil, Guayaquil, Ecuador.

Docente de la Facultad de Ciencias Matemáticas, Universidad de Guayaquil, Guayaquil, Ecuador. 


\section{Resumen}

La condición del Estero Salado en el Ecuador es uno de los principales problemas que preocupa a la humanidad. Existen estudios realizados por diferentes entidades sobre las características físicas, químicas, biológicas y económicas del mismo, con cuyos resultados se considera tomar medidas de mitigación del impacto generado por la contaminación del estero.

Esta investigación se enfoca en la caracterización de parámetros físicos - químicos en las cuencas de drenaje del Estero Salado en la ciudad de Guayaquil, tiene como objetivo principal de determinar la variación de concentración de cada parámetro con respecto al sistema de marea y estaciones climáticas del agua y sedimentos del estuario.

La toma de muestra es realizada en cuatros puntos del Estero, tomando en cuenta los métodos de muestreo respectivos. Los parámetros analizados en las muestras de agua son $\mathrm{OD}, \mathrm{pH}$, Temperatura, Salinidad, DBO, DQO, Cd, Cu, Hg, Pb, Zn, Mn, NT, NH3-N, NO2, NO3, F y Coliformes fecales y en las muestras de sedimentos fueron $\mathrm{Cd}, \mathrm{Cu}, \mathrm{Hg}, \mathrm{Pb}$ y $\mathrm{Zn}$.

Los resultados de la caracterización fueron comparados con la normativa ambiental vigente y de acuerdo al análisis de resultados se recomienda un plan para minimizar la contaminación en el estero.

Palabras claves: Estero Salado, estuario, caracterización físico-químico de Estero Salado. 


\section{Abstract}

The condition of the Salt Estuary in Ecuador is one of the main problems that concern humanity. There are studies carried out by different entities on the physical, chemical, biological and economic characteristics of the same, whose results are considered to take measures to mitigate the impact generated by the pollution of the estuary.

This research focuses on the characterization of physical - chemical parameters in the drainage basins of the Estero Salado in the city of Guayaquil, whose main objective is to determine the concentration variation of each parameter with respect to the tidal system and climatic seasons of the water And estuarine sediments.

Sampling is carried out in four points of Estero, taking into account the respective sampling methods. The parameters analyzed in the water samples are OD, $\mathrm{pH}$, Temperature, Salinity, BOD, $\mathrm{COD}, \mathrm{Cu}, \mathrm{Hg}, \mathrm{Pb}, \mathrm{Zn}, \mathrm{Mn}, \mathrm{NT}, \mathrm{NH} 3-\mathrm{N}, \mathrm{NO} 2, \mathrm{NO} 3, \mathrm{~F}$ and Fecal Coliforms. Sediment samples were $\mathrm{Cd}, \mathrm{Cu}, \mathrm{Hg}, \mathrm{Pb}$ and $\mathrm{Zn}$.

The results of the characterization were compared with the current environmental regulations and according to the analysis of results a plan to minimize pollution in the estuary is recommended.

Key words: Estero Salado, estuary, physical-chemical characterization of Estero Salado 


\section{Resumo}

A condição do Estero Salado no Equador é um dos principais problemas de interesse para a humanidade. Estudos realizados por diferentes entidades sobre as características químicas, físicas, biológicas e econômicas, com os resultados a considerar tomar medidas para mitigar o impacto causado pela poluição do estuário.

Esta investigação centra-se na caracterização de parâmetros físico - químicos nas áreas de captação de Estero Salga Guayaquil, principal objetivo de determinar a mudança na concentração de cada parâmetro no que diz respeito ao sistema de estações meteorológicas maré e água e estuários sedimentos.

A amostragem é realizada em quatro pontos de estero, tendo em conta os respectivos métodos de amostragem. Os parâmetros analisados em amostras de água são $\mathrm{OD}, \mathrm{pH}$, temperatura, salinidade, BOD, COD, Cd, Cu, Hg, Pb, Zn, Mn, NT, N-NH3, NO2, NO3, F e coliformes fecais e o amostras de sedimentos foram $\mathrm{Cd}, \mathrm{Cu}, \mathrm{Hg}, \mathrm{Pb}$ e $\mathrm{Zn}$.

Os resultados da caracterização foram comparados com corrente e de acordo com análise de regulamentos ambientais resulta um plano para minimizar a poluição no estuário é recomendado.

Palavras-chave: Matting Sal, estuário, caracterização físico-química Matting Sal 


\section{Introducción.}

La preservación de la integridad de las fuentes de agua implica conservar el balance natural de sus condiciones físicas, químicas y biológicas como un todo,

Sin embargo, el mismo se encuentra alterando el ecosistema acuático (Reche, 2003)por el incremento de los desechos propios de las actividades humanas.

En el Ecuador los manglares forman parte de una variedad de hábitats que se extienden a lo largo de aproximadamente $2900 \mathrm{~km}$ de línea de costa (Boothroyd et al, 1994), siendo uno de los estuarios de mayor importancia en el Estero Salado por formar parte del Golfo de Guayaquil, situado en la provincia del Guayas y representado por seis especies de mangle, de los cuales tres son conocidos comúnmente como mangles rojos Rhizophora mangle, R. harrisonii, R. racemosa, Conocarpus erectus (mangle jelí), Laguncularia racemosa (mangle blanco) (Valverde y Pérez, 2012) y Avicennia germinans (mangle negro) (Pérez, 2012), así como otras formaciones vegetales hidrohalófitas (ej. Salicornia fruticosa, Cryptocarpus pyriformes, Batis maritima). Las aguas del Golfo son biológicamente fértiles y soportan importantes pesquerías artesanales del Golfo de Guayaquil como las de peces como el barrilete (Katsuwonus pelamis), aleta amarilla (Thunnus albacares) (Stevenson, 1981); moluscos como Ostrea columbiensis (Mora y Reinoso, 1981), Anadara tuberculosa, A. similis, Mytella guyanensis, M. strigata; y crustáceos como el cangrejo Ucides occidentalis.

El Golfo de Guayaquil, es considerado el estuario más grande de la costa sudamericana del Pacífico. Posee una extensión de 13701 km2 (11711 km2 de superficie de agua y 1990 km2de islas e islotes) (CAAM, 1996; Stevenson, 1981). El Golfo está dividido naturalmente en estuario exterior e interior. El Estero Salado y el sistema del río Guayas forman parte del estuario interior, el Estero 
Salado se inicia en la ciudad de Guayaquil y se extiende hacia el Sur-Oeste, hasta el Canal del Morro (Cruz, 1992) y tiene una extensión de 74 km (Stevenson, 1981).

Sin embargo, por incursionar en la ciudad más poblada del Ecuador (Valverde, F. D. y J. Pérez 2012), el Estero Salado está siendo afectado en diferentes formas por las actividades antropogénicas que se realizan en la urbe y en sus alrededores. Así tenemos el crecimiento poblacional en sus riberas, residuos sólidos que son desechados de manera inadecuada, desarrollo industrial, descargas de aguas residuales que no cumplen con los límites permisibles o a la falta de educación ambiental que tienen los habitantes periféricos del estero al arrojar desperdicios hacia el mismo. Por lo tanto, dicha contaminación tiene una repercusión muy elevada sobre todas las variables naturales y sociales.

El Estero Salado, por ser un brazo de mar y al no recibir aportes de afluentes o ríos situados aguas arriba, sus aguas tienen cierto movimiento que no está dirigido predominante hacia el mar abierto; el cuerpo de agua de desliza con la marea hacia el mar, pero recupera su posición inicial con el reflujo de la misma; este comportamiento afecta al proceso de renovación y autodepuración de las aguas en el Estero Salado, especialmente hacia la zona que delimita con la ciudad de Guayaquil. (Holland, A.F., Sanger, D.M., Gawle, C.P., Lerberg, S.B., Santiago., M.S., Riekerk, G.H.M., Zimmerman, L.E., Scott, G.E., 2004)

Este efecto, sumado año tras año, ha influenciado de una u otra manera al deterioro de la calidad de ambiental y estética paisajística del Estero, afectando al número de especies, abundancia y tamaño de organismos de importancia ecológica y comercial que habitan en el estero. 
Por este motivo es necesario determinar las condiciones físico-químico (calidad de agua y sedimento) del Estero Salado y su influencia en el medio ambiente mediante un análisis de la calidad y la comparación de las normas ambientales actuales del Ecuador.

\section{Materiales y métodos.}

\section{AREA DE ESTUDIO}

El Estero Salado es un sistema estuarino situado al occidente del río Guayas y al Oeste del Golfo de Guayaquil, Ecuador, el cual se ramifica hasta la ciudad de Guayaquil, el presente trabajo se realizó en el tramo $\mathrm{B}$, el cual es denominado así por las autoridades de control ambiental de la ciudad.

Las estaciones que se monitorearon fueron ubicadas en los siguientes puntos: Puente las Monjas, Centro Comercial Albán Borja, Federación Deportiva del Guayas y Mi Comisariato Mapasingue este. El trabajo se realizó durante un año en los meses de agosto $(30$ y 31 de agosto del 2015) y el (8 de diciembre del 2015), es decir entradas de cada estación invierno y verano, con el objetivo de comparar la variación de que existe en cada una. Los muestreos se realizaron en estado de marea de Sicigia, en diferentes horas de luminosidad. El estado de marea fue el siguiente:

30 de agosto del 2015: 07:34 pleamar; 4,31 altura de metros y 14:40 bajamar; -0,01 altura en metros.

31 de agosto del 2015: 08:24 pleamar; 4,38 altura de metros y 15:28 bajamar; -0,09 altura en metros.

8 de diciembre del 2015: 17:40 pleamar; 3,42 altura de metros y 12:04 bajamar; 0,20 altura en metros. 


\begin{tabular}{|c|c|c|c|c|}
\hline \multirow{2}{*}{ Lugar } & \multirow{2}{*}{\multicolumn{2}{|c|}{ Sectores }} & \multicolumn{2}{|c|}{ Coordenadas UTM } \\
\hline & & & Norte & Este \\
\hline \multirow{4}{*}{$\begin{array}{l}\text { Estero Salado de la } \\
\text { ciudad de Guayaquil, } \\
\text { Ecuador }\end{array}$} & \multirow{4}{*}{$\begin{array}{lr}\text { Cuencas } & \text { de } \\
\text { drenaje } & \text { del } \\
\text { Tramo B } & \end{array}$} & Puente Las Monjas & 9759866 & 620910 \\
\hline & & Centro Comercial Albán Borja & 9760420 & 620544 \\
\hline & & Federación Deportiva del Guayas & 9761029 & 619987 \\
\hline & & Mi Comisariato Mapasingue Este & 9761239 & 619258 \\
\hline
\end{tabular}

Cuadro 1: coordenadas de los puntos de muestreo de agua y sedimento del estero salado en el Ecuador

Se seleccionaron 4 estaciones de muestreo (P1, P2, P3 y P4) a fin de determinar el efecto de la calidad de agua y sedimento en áreas de asentamiento humanos provistos de servicios de agua potable, alcantarillado y servicios urbanísticos. Las coordenadas de cada muestreo fueron determinadas utilizando un GPS modelo Etrex garmin y se muestran en el cuadro I.

En cada estación se captó muestras de agua superficial y sedimentos, en estado de marea de Sicigia, en diferentes horas de luminosidad, el agua se recolecto en envases plásticos esterilizados de un $\mathrm{L}$ y el sedimento en fundas esterilizadas para $1 \mathrm{Kg}$, los cuales estaban previamente identificados. Una vez recolectadas las muestras se pusieron en una hielera para mantener temperatura y Luego esas muestras fueron llevadas a dos laboratorios (Químicos Marcos y Universidad de Guayaquil) para su posterior análisis.

La metodología de muestreo para agua es realizada en base a la Normas Técnica Ecuatoriana (NTE):

NTE INEN 2176: 1998. Agua. Calidad del Agua. Muestreo. Técnicas de Muestreo.

NTE INEN 2169: Agua. Calidad del agua. Muestreo. Manejo y conservación de muestras.

Para muestreo del sedimento: 
NTE INEN ISO 5667-15:2006: Calidad del agua. Muestreo. Parte 1: Guía para el diseño de los programas de muestreo y técnicas de muestreo.

Los parámetros para los análisis físicos - químicos se realizaron en situ y en laboratorios, estos son temperatura, salinidad y oxígeno disuelto(O D), que fueron tomados en situ y los analizados en los laboratorios son demanda química de oxígeno (DQO), Manganeso (Mn), Nitrógeno Total (NT), Nitrógeno Amoniacal $\left(\mathrm{NH}_{3}-\mathrm{N}\right)$, Nitrato $\left(\mathrm{NO}_{2}\right)$, Nitrito $\left(\mathrm{NO}_{3}\right)$, Flúor $(\mathrm{F})$, Cadmio (Cd), Cobre ( $\mathrm{Cu}$ ), Mercurio (Hg), Plomo (Pb), Cinc ( $\mathrm{Zn})$, demanda bioquímica de oxígeno (DBO), y Coliformes fecales.

Para la medición de las muestras de agua in situ se usaron los equipos Waterproof Pen PH Test para medir $\mathrm{pH}$ y temperatura, Waterproof Salt Test para medir el nivel de salinidad y Jenway 970 DO2 Meter para medir el Oxígeno Disuelto. Los análisis realizados de agua en el laboratorio de la Universidad de Guayaquil fueron medidos con el equipo HACH DR2800 mediante los métodos descritos en el cuadro II. Y en el laboratorio Químicos Marcos los métodos analíticos para el análisis de agua en Cadmio, Cobre, Mercurio, Plomo, Cinc es PEE-GQM-FQ-33, para la demanda bioquímica de oxígeno es PEE-GQM-FQ-05, Coliformes fecales es 9222 D y para suelo el Cadmio, Cobre y Cinc el método analítico es 3120 B, y para cobre y mercurio es PEE-GQM-FQ-54. Todos estos parámetros fueron comparados con la Normativa Ambiental vigente, Acuerdo Ministerial No. 97 R.O. 30 de julio 2015.

\section{Resultados.}

En el análisis de resultados se hace la comparación con los límites máximos descritos en la Normativa Ambiental vigente el Acuerdo Ministerial 097-A: 
Se compara la variación de cada uno de los parámetros con respecto a los horarios de mareas y estaciones climáticas.

\section{Caracterización del Agua del Estero Salado}

Los cuadros II y III muestran los parámetros analizados en donde se observa que el $\mathrm{OD}, \mathrm{NO}_{2}$ y F se encuentran por debajo del límite máximo. Los parámetros $\mathrm{NO}_{3}, \mathrm{Hg}, \mathrm{Zn}, \mathrm{Mn}, \mathrm{NH}_{3}-\mathrm{N}$ superan totalmente el límite máximo. El pH se encuentra entre el rango óptimo.

El Cd supera los límites máximos en bajamar en el mes de agosto (Ap1 y Ap2); en pleamar en agosto - Ap1 y en diciembre (Ap1 y Ap2). El Cu, en agosto - Ap1 y diciembre - Ap4 en pleamar y en bajamar en agosto - Ap2 superan el límite máximo. $\mathrm{DBO}_{5}$, en pleamar en agosto (Ap3 y Ap4); en bajamar en agosto (Ap1, Ap2 y Ap4) superan los límites máximos. La DQO en bajamar en agosto (Ap3 y Ap4) y diciembre (Ap2 y Ap3) se encuentran por debajo de los límites máximos permitidos. Los coliformes fecales en - Ap1 en pleamar y bajamar se encuentran por debajo de límite máximo.

\section{Caracterización del Suelo del Estero Salado}

Los cuadros IV y V muestran la caracterización de suelo del Estero Salado en las cuencas de drenaje del tramo B, en los horarios de pleamar y bajamar y en las dos estaciones climáticas del año en donde se observa que todos los parámetros monitoreados superan los límites máximos descritos en la normativa ambiental. 
Evaluación de la calidad físico-químico en agua y sedimentos del estero salado en el noroeste de América Latina

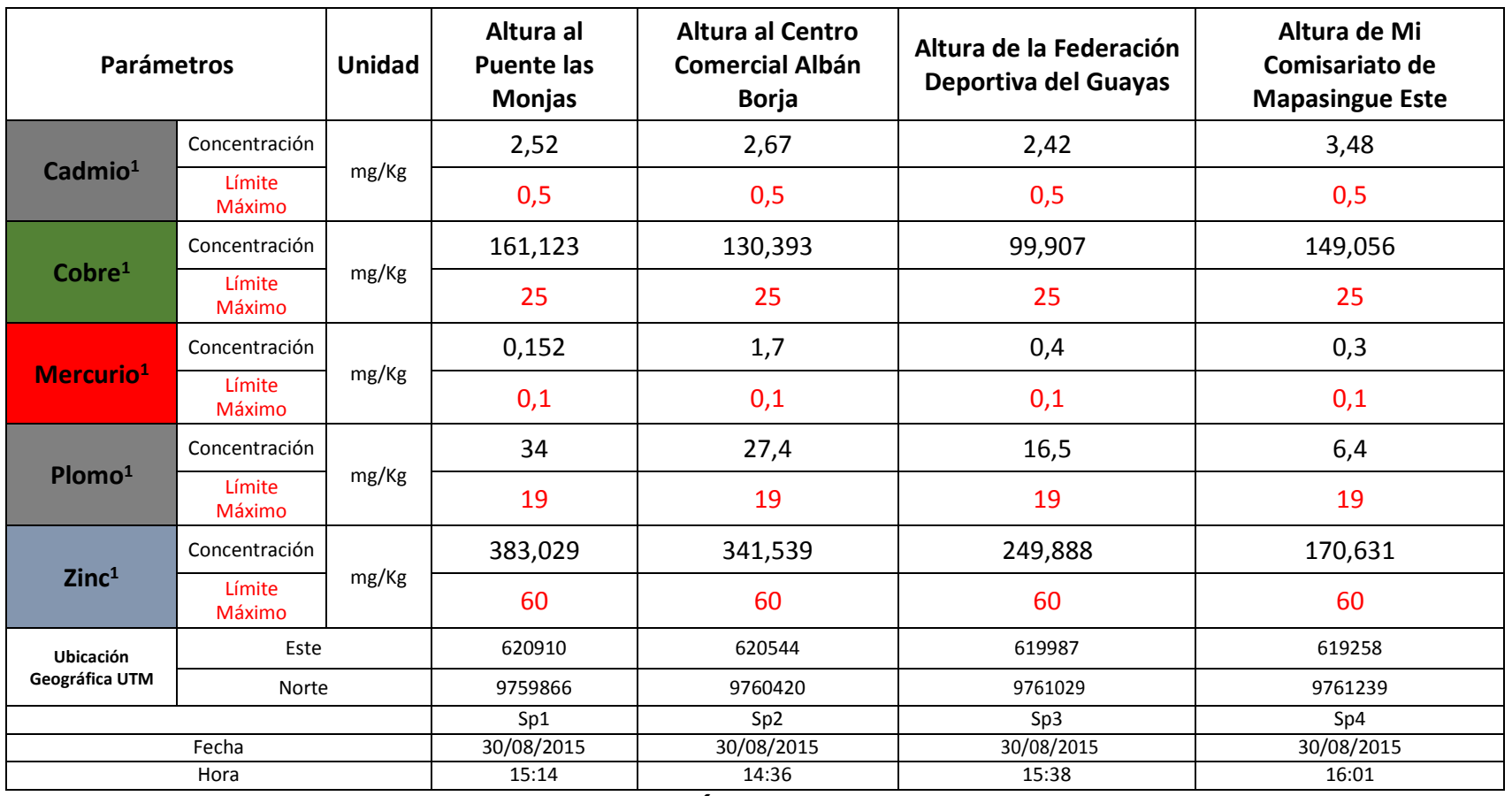

\section{CUADRO IV: RESULTADOS DE CARACTERIZACIÓN DE SUELO DEL ESTERO SALADO EN LA CUENCA DE} DRENAJE DEL TRAMO B - AGOSTO DEL 2015

1 Acuerdo Ministerial No. 97 R.O. 30 de julio 2015, anexos del Texto Unificado de Legislación Secundaria del Ministerio del Ambiente reformado mediante Acuerdo Ministerial No. 61. Anexo 2, tabla 1. Criterios de calidad de suelo.

** Análisis realizado por laboratorio Grupo Químico Marcos

\begin{tabular}{|c|c|c|c|c|c|c|}
\hline \multicolumn{2}{|c|}{ Parámetros } & Unidad & $\begin{array}{l}\text { Altura al } \\
\text { Puente las }\end{array}$ & $\begin{array}{l}\text { Altura al Centro } \\
\text { Comercial Albán }\end{array}$ & Altura de la Federación & $\begin{array}{c}\text { Altura de Mi } \\
\text { Comisariato de }\end{array}$ \\
\hline \multirow{2}{*}{ Cadmio $^{1}$} & Concentración & \multirow{2}{*}{$\mathrm{mg} / \mathrm{Kg}$} & 2,1875 & 1,9029 & 1,889 & 1,9767 \\
\hline & $\begin{array}{l}\text { Límite } \\
\text { Máximo }\end{array}$ & & 0,5 & 0,5 & 0,5 & 0,5 \\
\hline \multirow{2}{*}{ Cobre $^{1}$} & Concentración & \multirow{2}{*}{$\mathrm{mg} / \mathrm{Kg}$} & 185,32 & 103,625 & 175,214 & 95,946 \\
\hline & $\begin{array}{l}\text { Límite } \\
\text { Máximo }\end{array}$ & & 25 & 25 & 25 & 25 \\
\hline \multirow{2}{*}{ Mercurio ${ }^{1}$} & Concentración & \multirow{2}{*}{$\mathrm{mg} / \mathrm{Kg}$} & 0,8 & 0,3 & $<0,3$ & 0,4 \\
\hline & $\begin{array}{l}\text { Límite } \\
\text { Máximo }\end{array}$ & & 0,1 & 0,1 & 0,1 & 0,1 \\
\hline \multirow{2}{*}{ Plomo $^{1}$} & Concentración & \multirow{2}{*}{$\mathrm{mg} / \mathrm{Kg}$} & 47,2 & 28,5 & 24 & 26,4 \\
\hline & $\begin{array}{l}\text { Límite } \\
\text { Máximo }\end{array}$ & & 19 & 19 & 19 & 19 \\
\hline \multirow{2}{*}{ Zinc $^{1}$} & Concentración & \multirow{2}{*}{$\mathrm{mg} / \mathrm{Kg}$} & 441,293 & 248,954 & 342,399 & 162,346 \\
\hline & $\begin{array}{l}\text { Límite } \\
\text { Máximo }\end{array}$ & & 60 & 60 & 60 & 60 \\
\hline \multirow{2}{*}{$\begin{array}{l}\text { Ubicación } \\
\text { Geográfica UTM }\end{array}$} & \multicolumn{2}{|l|}{ Este } & 620910 & 620544 & 619987 & 619258 \\
\hline & \multicolumn{2}{|c|}{ Norte } & 9759866 & 9760420 & 9761029 & 9761239 \\
\hline & Sp1 & Sp2 & $\mathrm{Sp} 3$ & Sp4 \\
\hline \multicolumn{3}{|c|}{ Fecha } & $30 / 08 / 2015$ & $30 / 08 / 2015$ & $30 / 08 / 2015$ & $30 / 08 / 2015$ \\
\hline \multicolumn{3}{|c|}{ Hora } & $15: 14$ & $14: 36$ & $15: 38$ & $16: 01$ \\
\hline
\end{tabular}

CUADRO V: RESULTADOS DE CARACTERIZACIÓN DE SUELO DEL ESTERO SALADO EN LA CUENCA DE

DRENAJE DEL TRAMO B - DICIEMBRE DEL 2015

1 Acuerdo Ministerial No. 97 R.O. 30 de julio 2015, anexos del Texto Unificado de Legislación Secundaria del Ministerio del Ambiente reformado mediante Acuerdo Ministerial No. 61. Anexo 2, tabla 1. Criterios de calidad de suelo.

** Análisis realizado por laboratorio Grupo Químico Marcos 


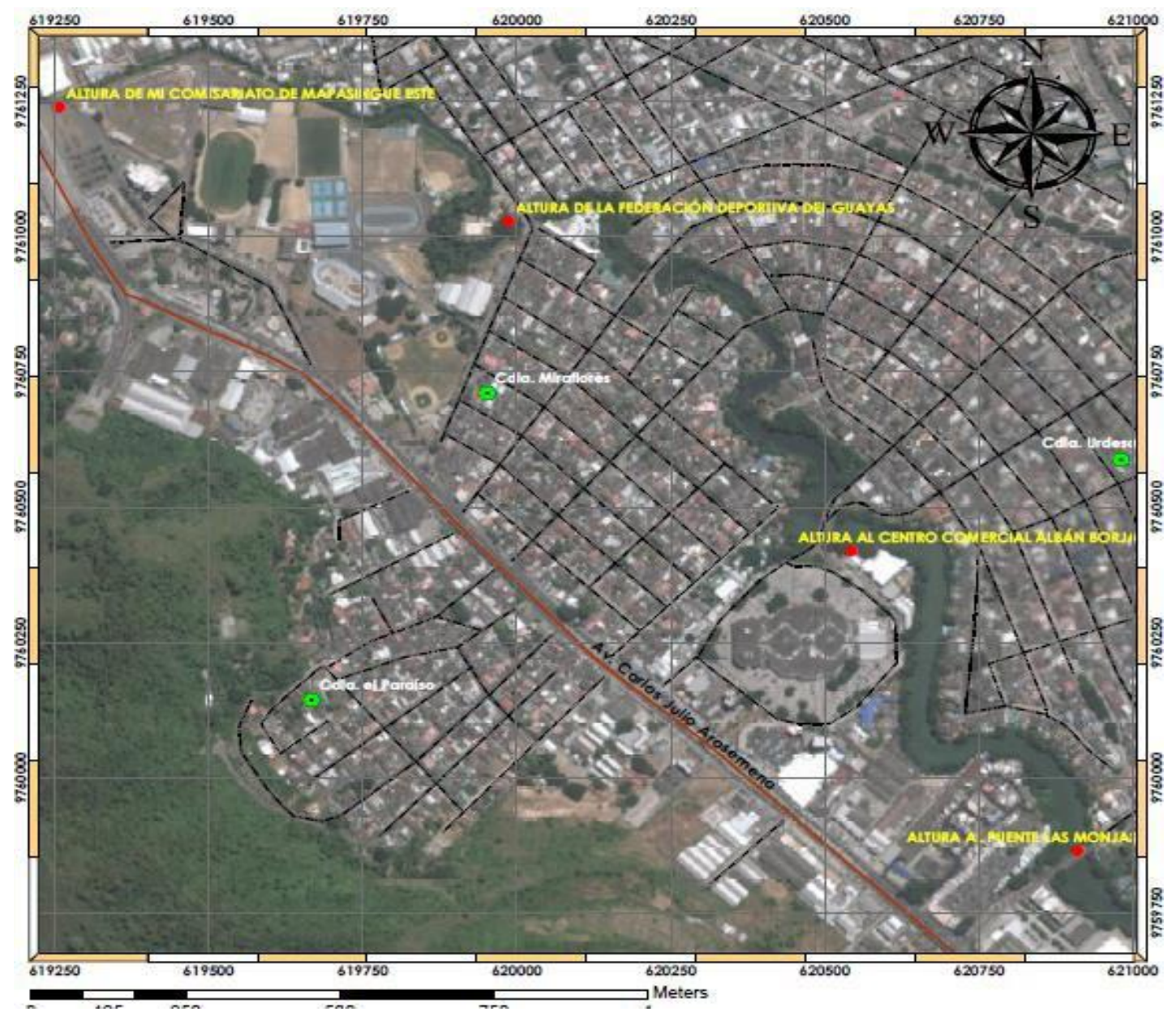

Fig. 1: Ubicación de los puntos de muestreos en el estero salado, Ecuador

\section{DISCUSION}

$A G U A$

Temperatura

Durante el mes de agosto del 2015 en las estaciones muestreados de pleamar la temperatura presentó un valor mínimo $22,20^{\circ} \mathrm{C}$ en las estaciones 1,2 y 4 y una máxima de $22,50^{\circ} \mathrm{C}$ en la estación 3, mientras que en el sistema de bajamar la mínima fue $21,80^{\circ} \mathrm{C}$ en el punto 3 y una 
máxima en la estación 1 con $23^{\circ} \mathrm{C}$, sin embargo en el mes de diciembre del 2015 en las estaciones muestreados de pleamar la temperatura presentó un valor mínimo $29^{\circ} \mathrm{C}$ en las estación 4 y una máxima de $31,10^{\circ} \mathrm{C}$ en la estación 2 , mientras que en el sistema de bajamar la mínima fue $29,70^{\circ} \mathrm{C}$ en el punto 4 y una máxima en la estación 1 con $32^{\circ} \mathrm{C}$. (fig. 2)

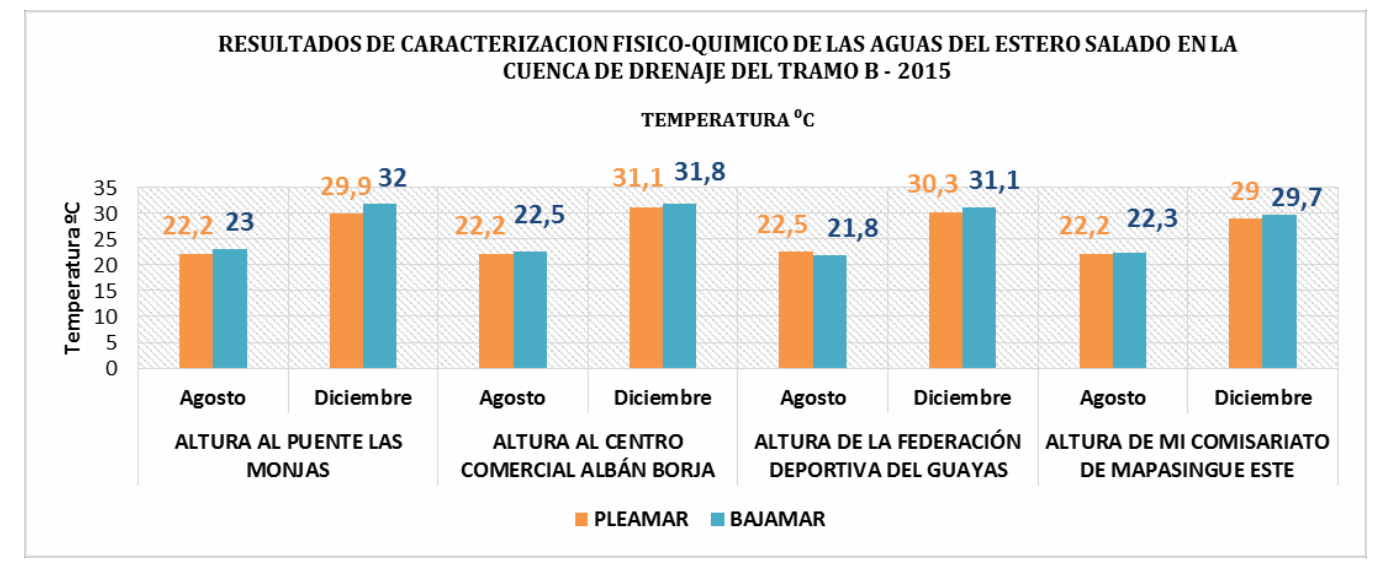

Fig. 2 Resultados de caracterización de la Temperatura en el agua del Estero Salado

La diferencia significativa de la temperatura durante los meses de agosto y diciembre es de $8,28^{\circ} \mathrm{C}$ con una temperatura promedio de $22,34^{\circ} \mathrm{C}$ para el mes de agosto y en diciembre 30,61 . Estos valores muestran que existió un incremento de temperatura hacia diciembre por ser el mes más cercano a la época cálida del año.

Este incremento sigue un ciclo anual, alterado principalmente por la ocurrencia del fenómeno del niño, por tal motivo dicho parámetro es un requerimiento esencial para el óptimo desarrollo de la biota estuarina y merece su evaluación.

Pudiendo causar efectos dañinos a la biota y reproducción de las especies, además de incrementar el crecimiento de bacterias y otros organismos que podrían reducir los niveles de oxígeno e incrementar el proceso de eutrofización en este ecosistema. 


\section{Salinidad}

La salinidad en el sistema de pleamar, se presentó con un valor mínimo de 0,3 UPS (Unidades porcentuales de salinidad) en la estación 4 y un valor máximo de 8,9 UPS en la estación 1 con un promedio de 4,12 UPS, mientras que en el sistema de bajamar el mínimo fue 0,3 UPS en las estaciones 3 y 4 y un máximo de 3,6 UPS en la estación 1 con un promedio de 0,96 UPS. (Fig3)

Cabe indicar que se observó que las aguas menos salinas ocurren en la estación 4 cuando estás ingresan al interior del estero con respecto a la estación 1, donde la salinidad se incrementa.

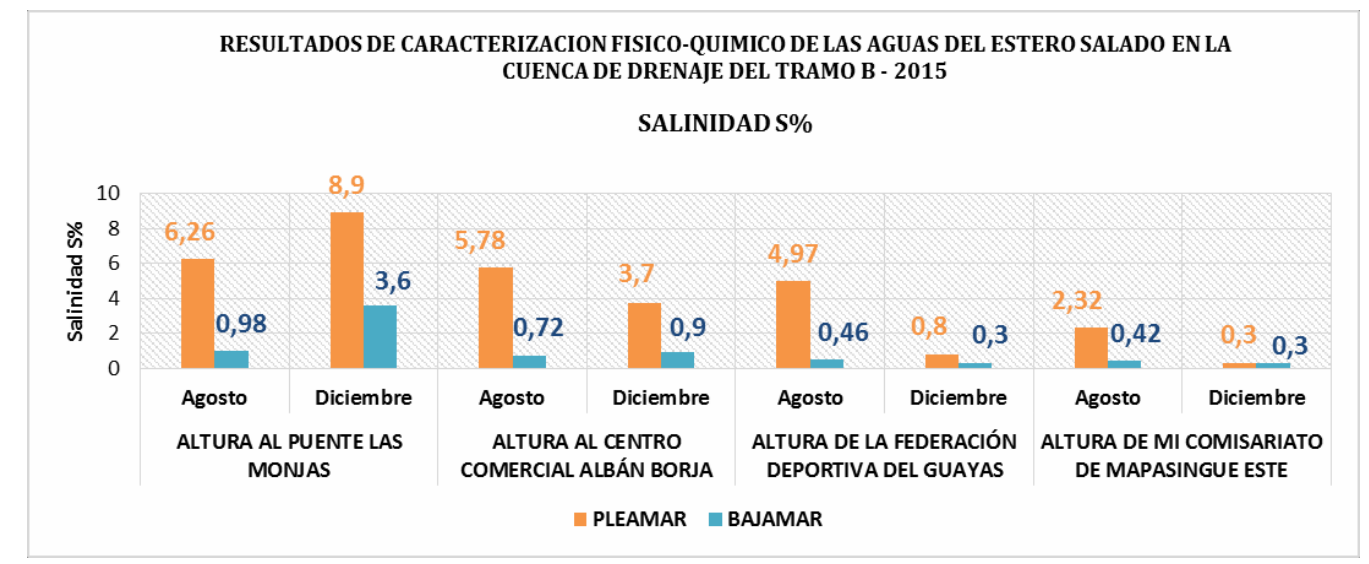

Fig. 3. Resultados de caracterización de Salinidad en el agua del Estero Salado

\section{Potencial Hidrógeno $(p H)$}

El pH en el sistema de pleamar presentó un valor mínimo 6,75 en la estación 1 y un valor máximo de 7,59 en la estación 3, con un promedio de 7,19 y un rango de 0.84 , mientras que en la bajamar el valor mínimo se presentó en 6,51 y el valor máximo fue de 7,61 en la estación 3, con un promedio de 7,06 y un rango de 1,1. (Fig.4) 


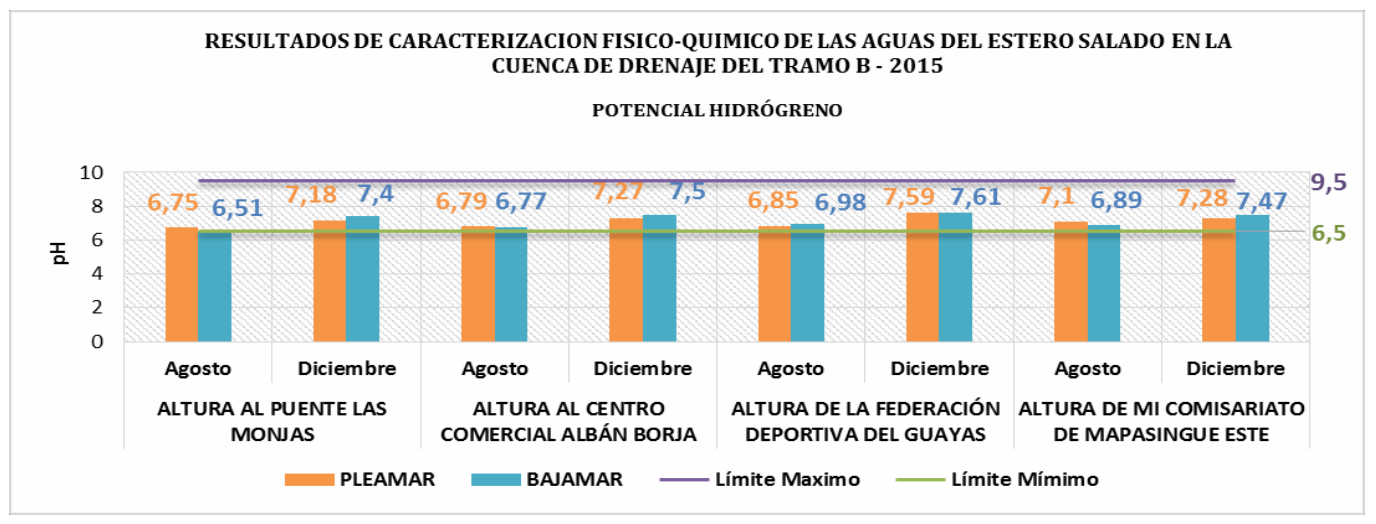

Fig.4. Resultados de caracterización del pH en el agua del Estero Salado

En los resultados obtenidos los valores se encuentran dentro del rango permisible para la preservación de la vida acuática y silvestre en las aguas de estuarios, esto es entre 6,5 y 9,5 lo anterior de acuerdo a la tabla 2 del anexo 1 del acuerdo ministerial No. 97.

\section{Oxígeno disuelto $(O D)$}

Los datos obtenidos del oxígeno disuelto presentaron valores muy inferiores a lo establecido por la normativa ambiental, presentando en pleamar un valor mínimo de $0,34 \mathrm{mgO}_{2} / 1$ y un máximo de 3,46 $\mathrm{mgO}_{2} / 1$, mientras que en la bajamar el valor mínimo se presentó en $0,33 \mathrm{mgO}_{2} / 1$ en la estación 3 y un máximo de $0,83 \mathrm{mgO}_{2} / \mathrm{l}$ en la estación 1. (Fig 5)

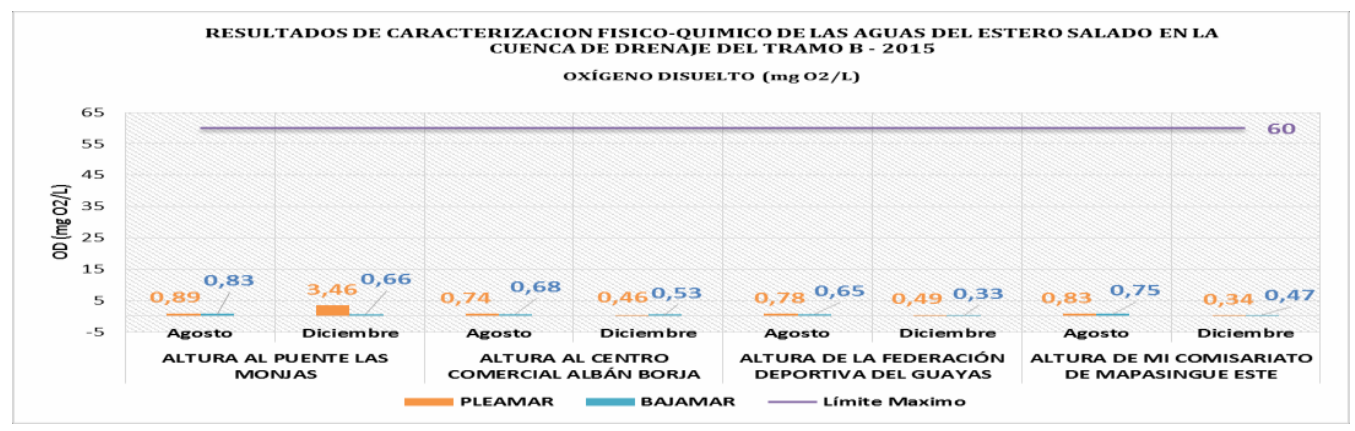

Fig. ¡Error! No hay texto con el estilo especificado en el documento.. Resultados de caracterización del Oxígeno Disuelto en el agua del 
Es importante indicar que, durante la pleamar, es decir, cuando se contempla el aporte de las aguas del flujo, se observa que la concentración de oxígeno disuelto es mayor que aquella presentada en la bajamar, ósea en el momento que se encuentra el más bajo nivel de aguas.

\section{$\operatorname{Mercurio}(\mathrm{Hg})$}

De acuerdo al análisis realizado los valores del mercurio se encuentran por encima de la normativa esto es $0,0001 \mathrm{mg} / \mathrm{l}$, es decir no cumplen con lo indicado en la tabla 2 del anexo 1 del acuerdo ministerial No. 97. (Fig. 6)

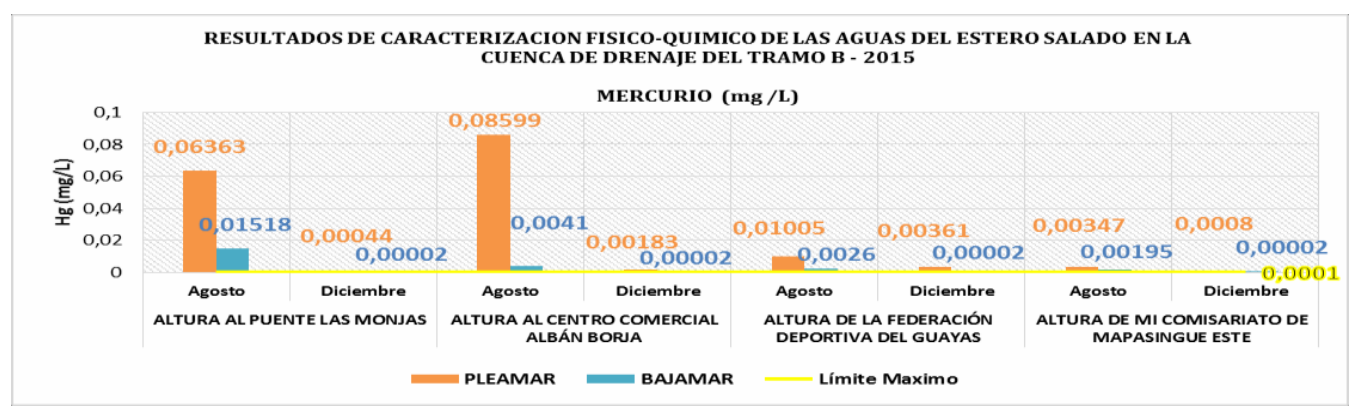

Fig. 6. Resultados de caracterización del Mercurio en el agua del Estero Salado

El mercurio presentó mayores concentraciones en pleamar en las estaciones 1 y 2 respectivamente, sin embargo, se puede diferenciar que en el mes de agosto los valores presentaron mayor incremento respecto al mes de diciembre.

\section{Cadmio $(C d)$}

El cadmio presentó concentraciones más altas en la estación 1,2 y 3 que sobrepasa los límites permisibles de la normativa ambiental $(0,05 \mathrm{mg} / \mathrm{l})$, en la estación 1 a la altura del puente las Monjas entre los meses de agosto y diciembre del 2015 presentaron los valores de 0,0165 mg/l y 0, $0096 \mathrm{mg} / \mathrm{l}$ y a la altura del centro comercial Albán Borja presentó durante la pleamar un valor de 
0,0431 mg/l y a la altura de la Federación Deportiva del Guayas presentó un valor de 0,0285 mg/l.

(Fig. 7)

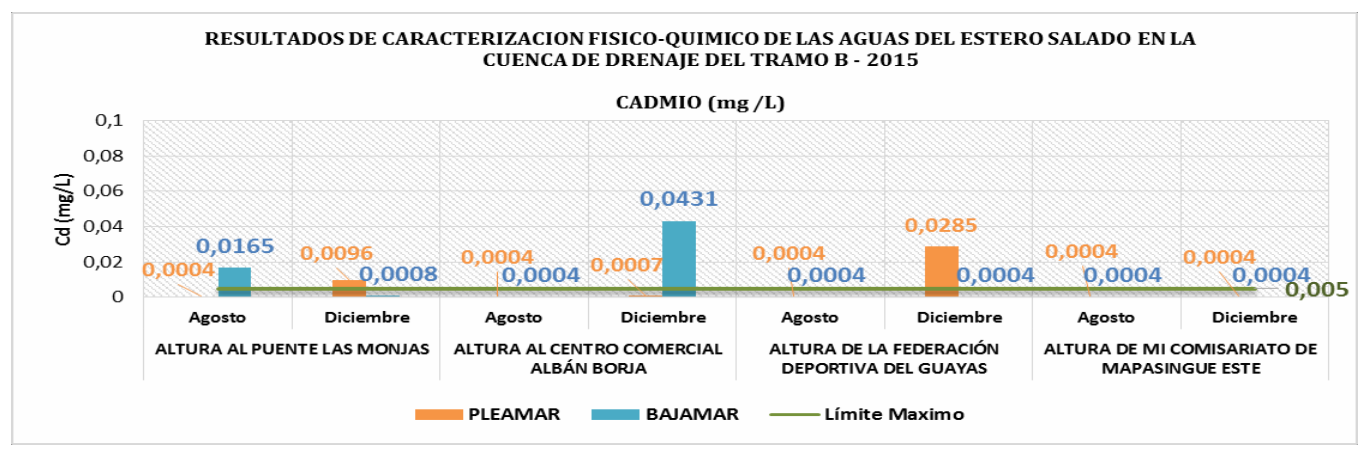

Fig. 7. Resultados de caracterización de Cadmio en el agua del Estero Salado

Cobre (Cu)

En el gráfico se observa que las concentraciones de cobre se encuentran más alta en las estaciones 1, 2 y 4 sobrepasando los límites permisibles de la normativa ambiental (0,005 mg/l), en la estación 1 y 2 a la altura del puente las Monjas y a la altura del centro comercial Albán Borja del mes de agosto del 2015 se presentaron en pleamar el valor de 0,238 mg/l y 0,0081 mg/l respectivamente, en la estación 4 en el mes de diciembre del 2015 a la altura de mi Comisariato de Mapasingue Este se puede evidenciar las concentraciones de 0,033 mg/l y 0,0155 mg/l entre pleamar y bajamar.

Presentó durante la pleamar un valor de 0,0431 mg/l y a la altura de la Federación Deportiva del Guayas presentó un valor de 0,0285 mg/l. (Fig. 8) 


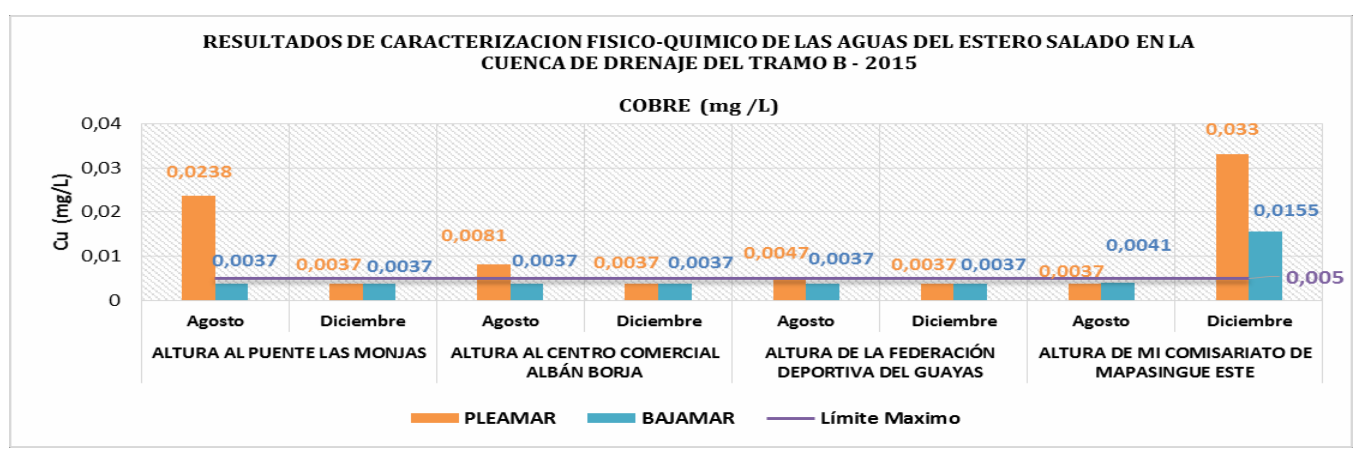

Fig. 8. Resultados de caracterización de Cobre en el agua del Estero Salado

Plomo $(\mathrm{Pb})$

De acuerdo al análisis realizado los valores de plomo se encuentran por encima de la normativa esto es $0,001 \mathrm{mg} / \mathrm{l}$, es decir no cumplen con lo indicado en la tabla 2 del anexo 1 del acuerdo ministerial No. 97. (Fig. 9)

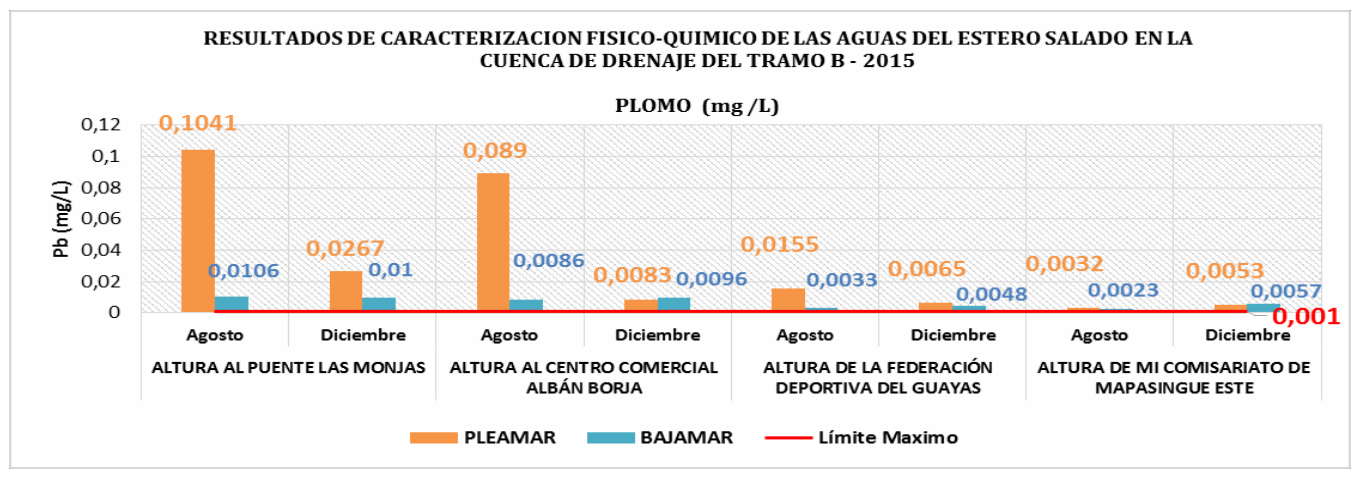

Fig. 9. Resultados de caracterización de Plomo en el agua del Estero Salado

$\operatorname{Cinc}(\mathrm{Zn})$

En la (fig. 10) se visualiza la concentración de Zn en agosto en pleamar, en las estaciones 1, 3 y 4 sobrepasa una pequeña cantidad del límite máximo, en comparación con los datos obtenidos en 
el mes de diciembre que sobrepasa en mayor cantidad el límite máximo (0,015 mg/L), esto indica que las aguas que ingresan contiene $\mathrm{Zn}$.

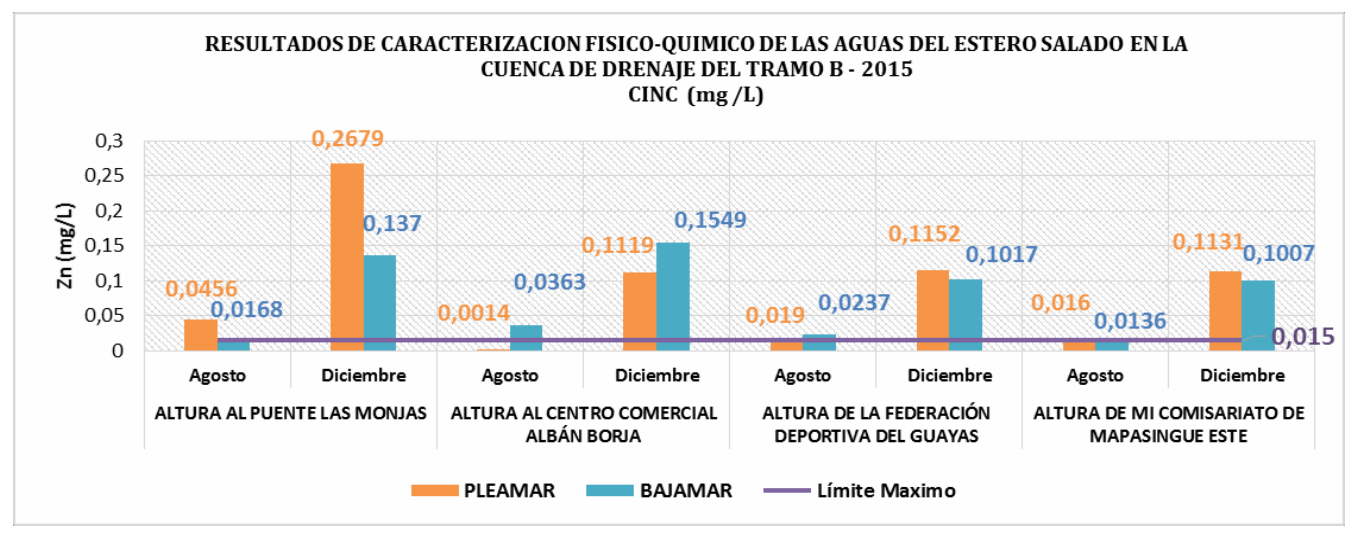

Fig. 10. Resultados de caracterización de Cinc en el agua del Estero Salado

\section{Demanda Bioquímica de Oxígeno $\left(\mathrm{DBO}_{5}\right)$}

La $\mathrm{DBO}_{5}$ en agosto en pleamar - estación 3 y 4, en bajamar - estación 1, 2 y 4 se observa que superan el límite máximo (20 mg/L); mientras que en diciembre se encuentra bajo el valor del mismo (fig. 11), debido a que en este mes comienza la época de invierno y existe una mayor oxigenación en el agua abasteciendo la demanda oxígeno necesaria para la biodegradación de la materia orgánica.

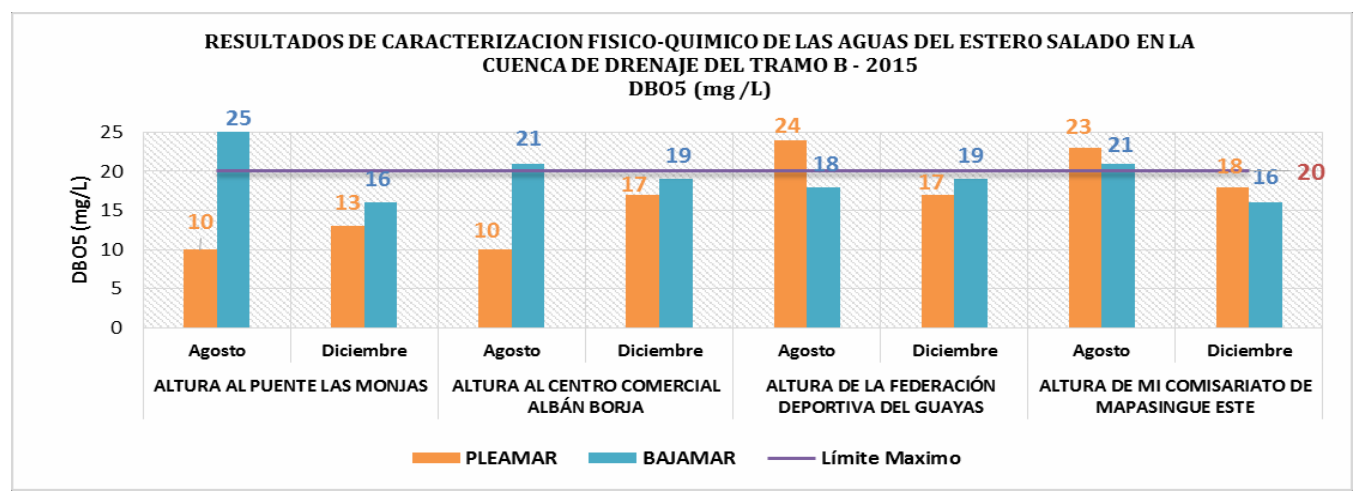

Fig. 11. Resultados de caracterización de la DBO5 en el agua del Estero Salado 
Demanda Química de Oxígeno (DQO)

En la (fig. 12) se observa que los valores de DQO en pleamar en la estación 1 en agosto y diciembre presenta una elevada demanda de oxígeno alcanzando $984 \mathrm{mg} / \mathrm{L}$ de $\mathrm{O}_{2}$, en comparación con las estaciones 2, 3 y 4 en ambos meses, excepto en agosto - estación 4, se visualiza valores entre 493 y $174 \mathrm{mg} / \mathrm{L}$ de $\mathrm{O}_{2}$, sin embargo, estos datos sobrepasan el valor óptimo que demanda la normativa ambiental. En bajamar en la estación 1 en agosto se determina un valor de $967 \mathrm{mg} / \mathrm{L}$ de $\mathrm{O}_{2}$, en las estaciones 2,3 y 4 los valores varían entre 13 y $591 \mathrm{mg} / \mathrm{L} \mathrm{deO} \mathrm{O}_{2}$.

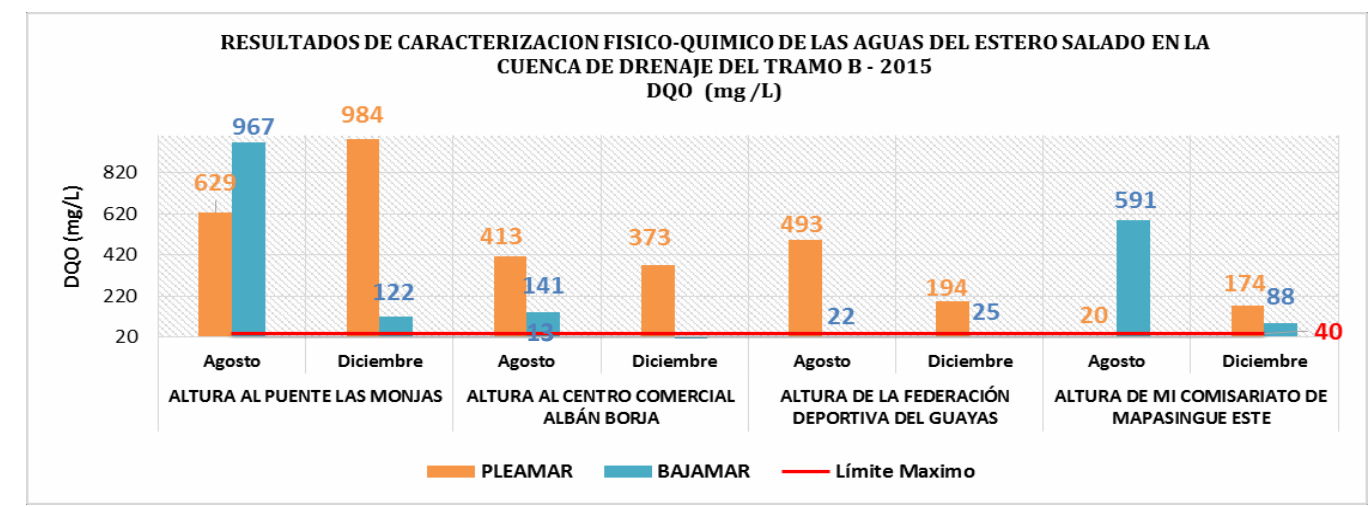

Fig. 12. Resultados de caracterización de la DQO en el agua del Estero Salado

Manganeso (Mn)

Los valores de Mn obtenidos en la caracterización del Estero Salado superan en límite máximo $(0,1 \mathrm{mg} / \mathrm{L})$ especificado por la normativa ambiental vigente los valores obtenidos desde 0,233 hasta $0,52 \mathrm{mg} / \mathrm{L}$ de este componente. (Fig 13) 


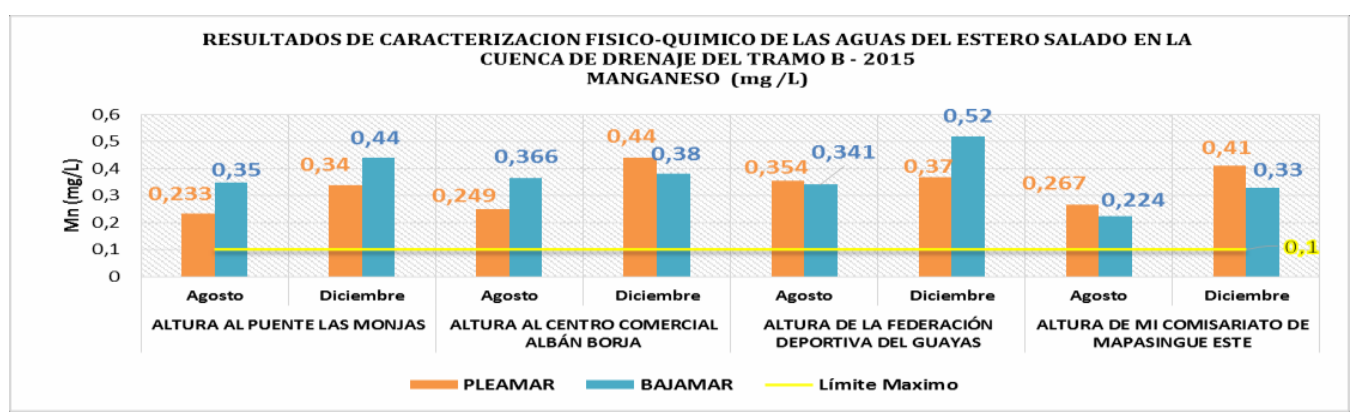

Fig. 13. Resultados de caracterización de Manganeso en el agua del Estero Salado

\section{Nitrógeno Total (NT)}

La cantidad de Nitrógeno en el agua del Estero presenta mayor concentración en pleamar, en el mes de agosto - estaciones 1 y 2 y en bajamar agosto - estación 4, en comparación con los otros puntos que se observa una concentración máxima de 104 (estación 1-bajamar-agosto) y mínima de 2,8 (estación 1-bajamar-diciembre). (Fig. 14)

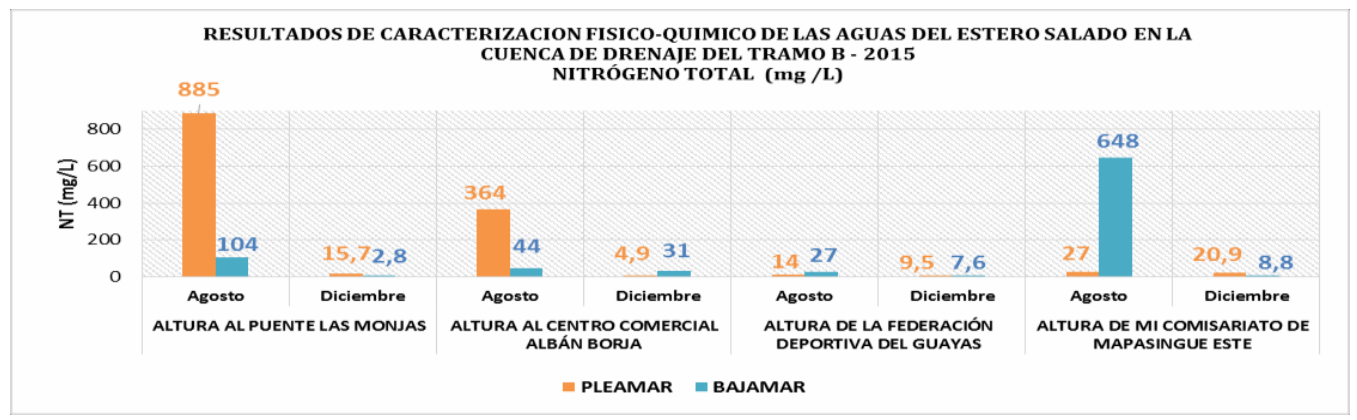

Fig. 14. Resultados de caracterización de Nitrógeno Total en el agua del Estero Salado

Nitrógeno Amoniacal $\left(\mathrm{NH}_{3}-\mathrm{N}\right)$

Los resultados obtenidos de la caracterización de $\mathrm{NH}_{3}-\mathrm{N}$ presente en el agua del estuario sobrepasan el límite máximo requerido $(0,4 \mathrm{mg} / \mathrm{L})$ por la normativa ambiental, las concentraciones varían de 6,44 (estación 2) hasta 16,72 (estación 4). (Fig 15) 


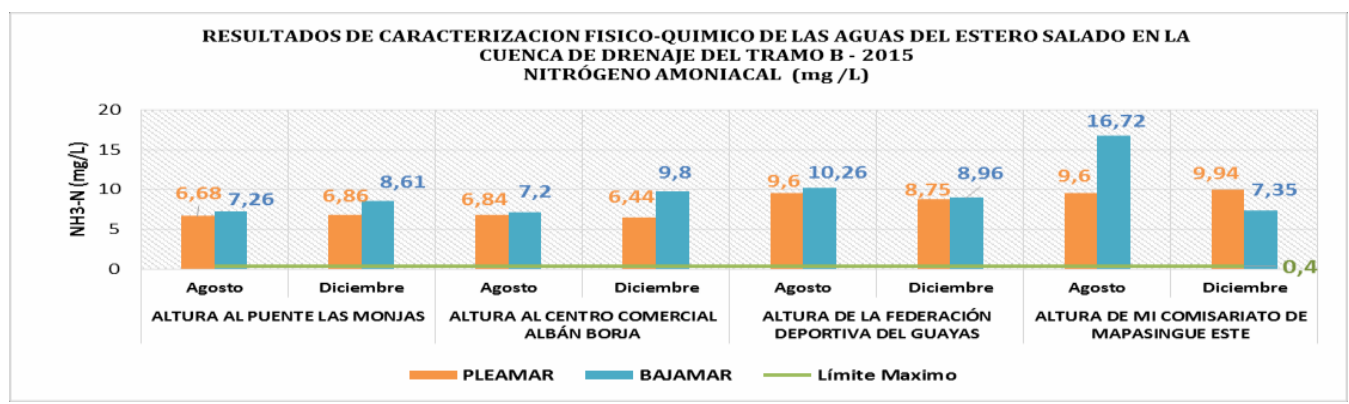

Fig. 15. Resultados de caracterización de Nitrógeno Amoniacal en el agua del Estero Salado

Nitrato $\left(\mathrm{NO}_{3}\right)$

En la (fig. 16) se observa que existe una deficiencia de Nitrato, el valor más alto es de 33,5 $\mathrm{mg} / \mathrm{L}$ en pleamar y $16 \mathrm{mg} / \mathrm{L}$ en bajamar presente a la altura de Mi comisariato de Mapasingue este, en las demás estaciones los valores fluctúan entre 6,6 y $0,2 \mathrm{mg} / \mathrm{L}$. en comparación con el límite máximo requerido que es de $200 \mathrm{mg} / \mathrm{L}$.

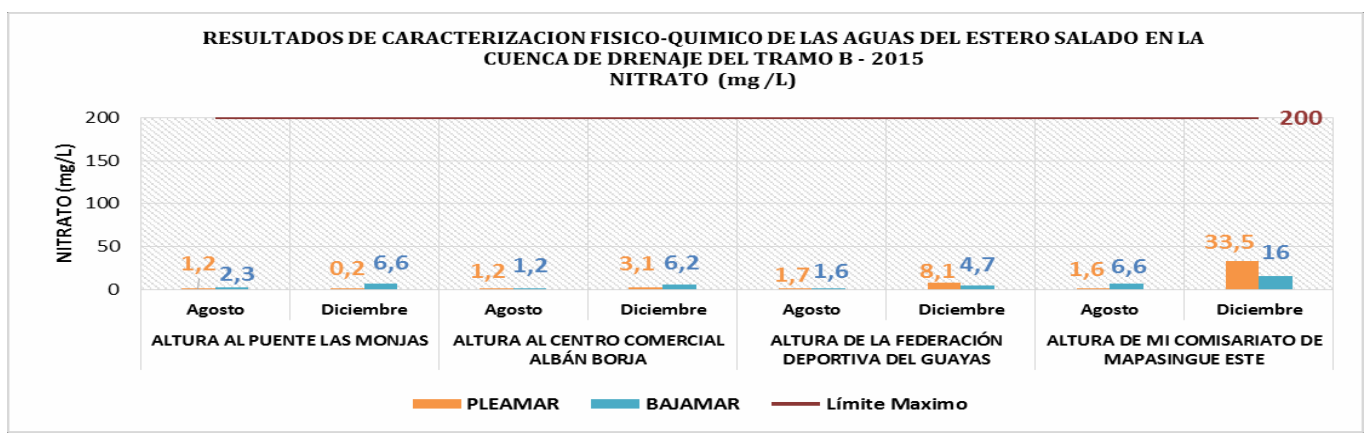

Fig. 16. Resultados de caracterización de Nitrato en el agua del Estero Salado

Nitrito $\left(\mathrm{NO}_{2}\right)$

Los datos de Nitrito obtenidos en la caracterización del estero superan el límite máximo $(0,2$ $\mathrm{mg} / \mathrm{L}$ ) determinado en la normativa ambiental, con un máximo de $22 \mathrm{mg} / \mathrm{L}$ en la estación 1 bajamar-diciembre y mínimo de 4,0 mg/L en la estación 2 -pleamar-agosto. (fig. 17) 


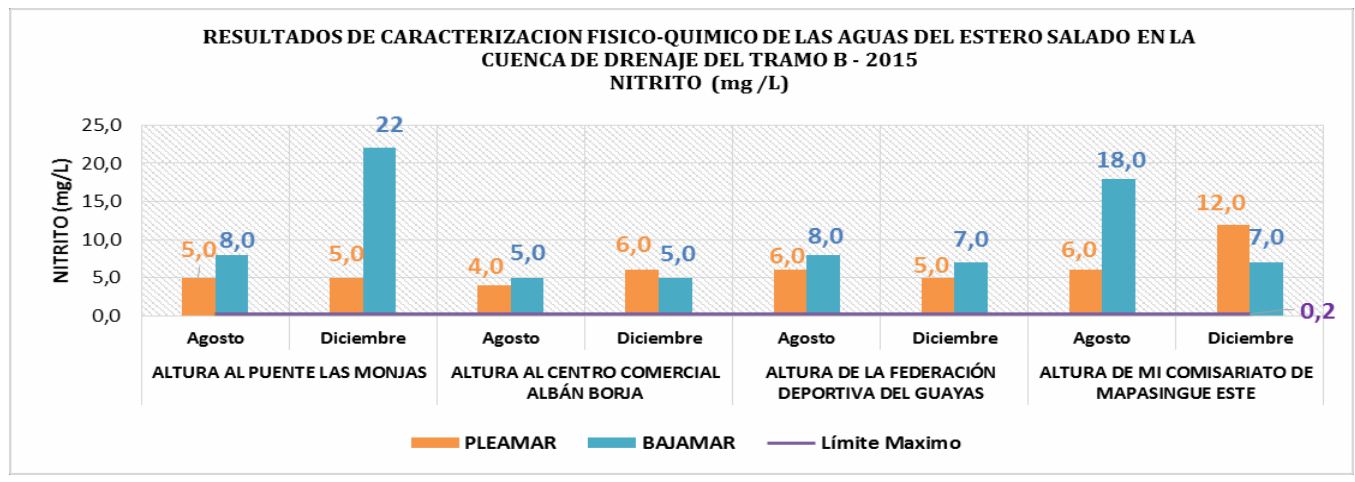

Fig. 17. Resultados de caracterización de Nitrito en el agua del Estero Salado

Flúor $(F)$

Los valores obtenidos de Fluor se encuentran cumpliendo con el límite máximo exigido por la normativa ambiental. Presentando una concentración desde 0,02 en la estación 3, hasta 1,03 en la estación 2. (Fig 18)

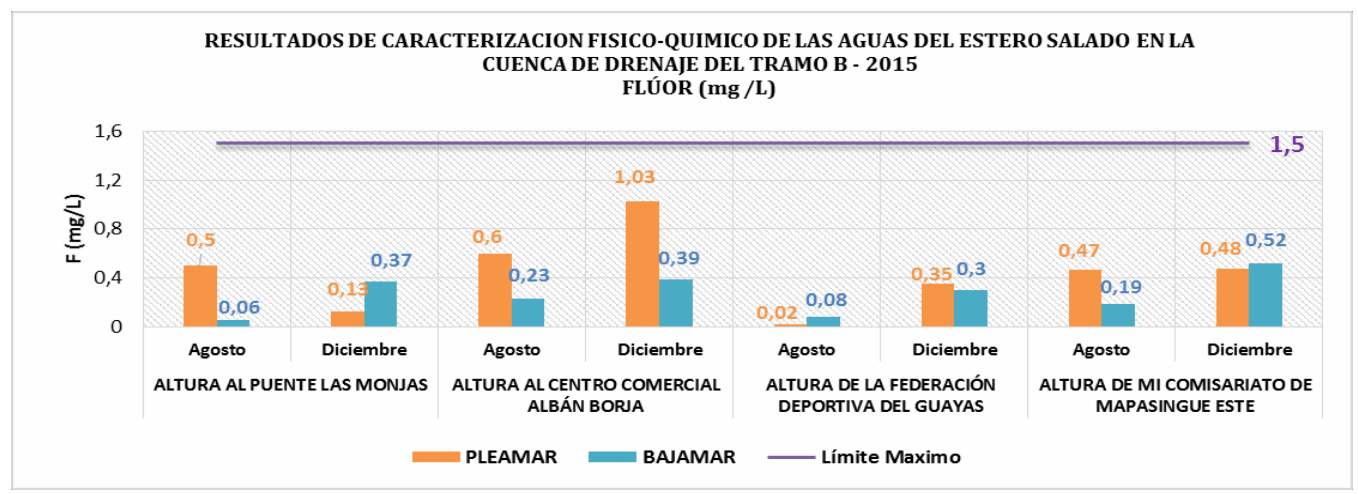

Fig. 18. Resultados de caracterización de Flúor en el agua del Estero Salado

\section{Coliformes Fecales}

El valor de los coliformes fecales sobrepasan el límite máximo (1000 NMP/100ml) determinado en la normativa ambiental vigente en todos los puntos muestreados; a excepción en diciembre en la estación 1, los resultados cumplen con la normativa. (Fig. 19) 


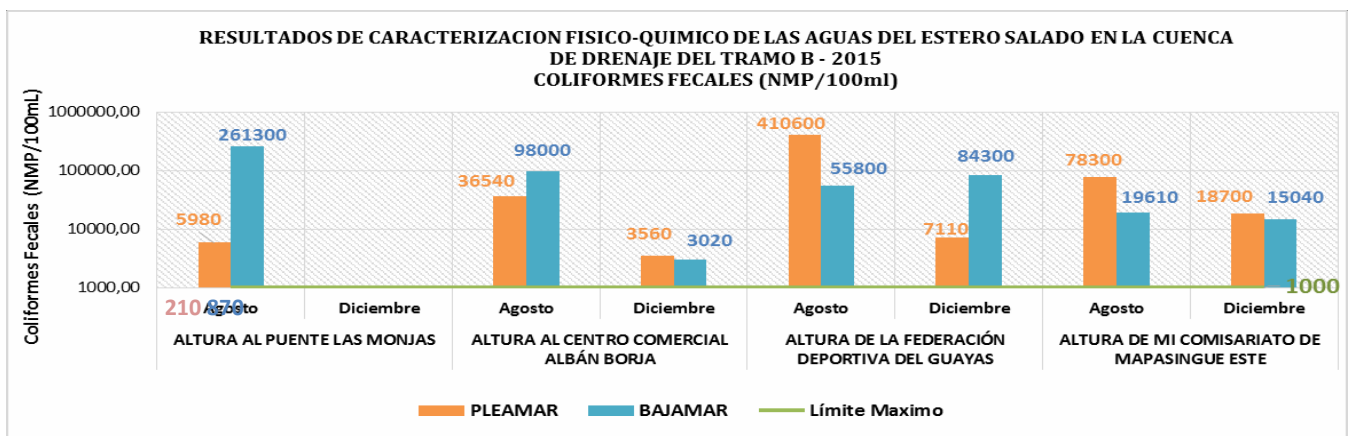

Fig. 19. Resultados de caracterización de Coliformes Fecales en el agua del Estero Salado SEDIMENTOS

Cobre (Cu)

Los resultados de la caracterización del sedimento del estero no cumplen con la normativa ambiental vigente, los valores fluctúan desde 95,946 hasta 185,32 mg/Kg de concentración de cobre, mientras que el valor máximo permitido es de $25 \mathrm{mg} / \mathrm{kg}$. (Fig. 20)

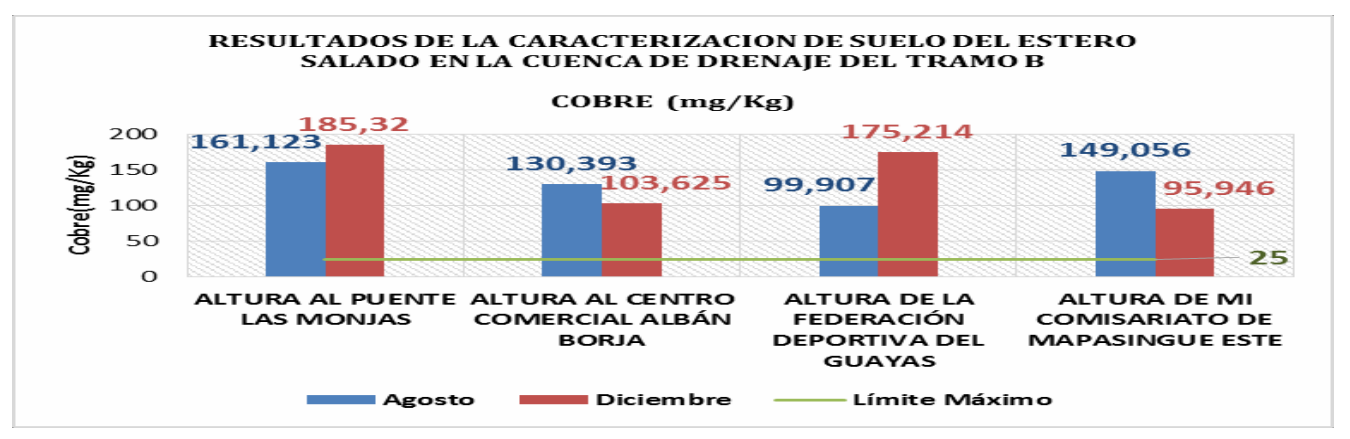

Fig. 20. Resultados de caracterización de Cobre en el sedimento del Estero Salado

\section{Cadmio $(C d)$}

Según la caracterización realizada la concentración de Cd en el sedimento del Estero Salado no cumple con el límite máximo $(0,5 \mathrm{mg} / \mathrm{Kg})$. Los valores fluctúan entre $1,889 \mathrm{mg} / \mathrm{Kg}$ en la estación 3 y 3,48 mg/ Kg en la estación 4 en el mes de agosto. (Fig. 21) 


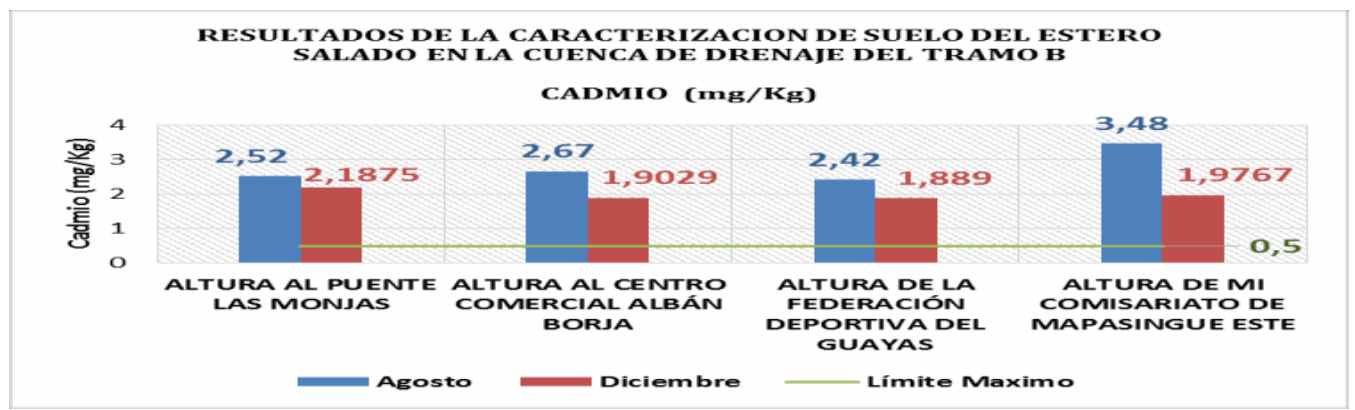

Fig. 21. Resultados de caracterización de Cadmio en el sedimento del Estero Salado

Cinc $(\mathrm{Zn})$

La (fig. 22) muestra que la concentración de Zn en el sedimento del estuario sobrepasa el límite máximo (60 mg/ Kg) determinado por la normativa ambiental vigente. Los valores varían desde 162,346 mg/ Kg en la estación 4 hasta 441,29 en la estación 1; ambos valores obtenidos en el mes de diciembre del 2015.

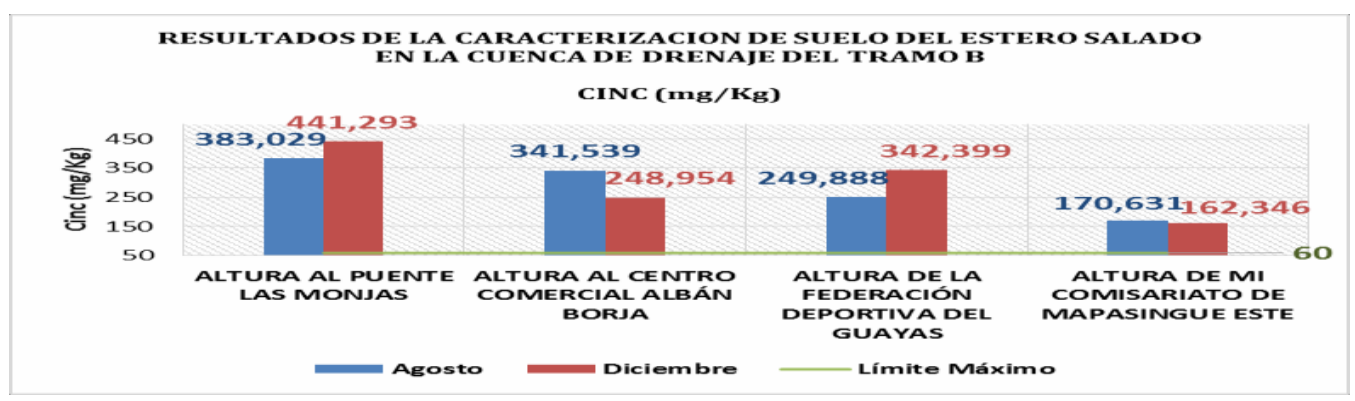

Fig. 22. Resultados de caracterización de Cinc en el sedimento del Estero Salado

$\operatorname{Mercurio}(\mathrm{Hg})$

La concentración de $\mathrm{Hg}$ contenida en el sedimento del Estero Salado no cumple con el límite máximo $(0,1 \mathrm{mg} / \mathrm{Kg})$ de acuerdo a los resultados de la caracterización. Los valores se presentan desde $0,152 \mathrm{mg} / \mathrm{Kg}$ en la estación 1 hasta $1,7 \mathrm{mg} / \mathrm{Kg}$ en la estación 2. (Fig. 23) 


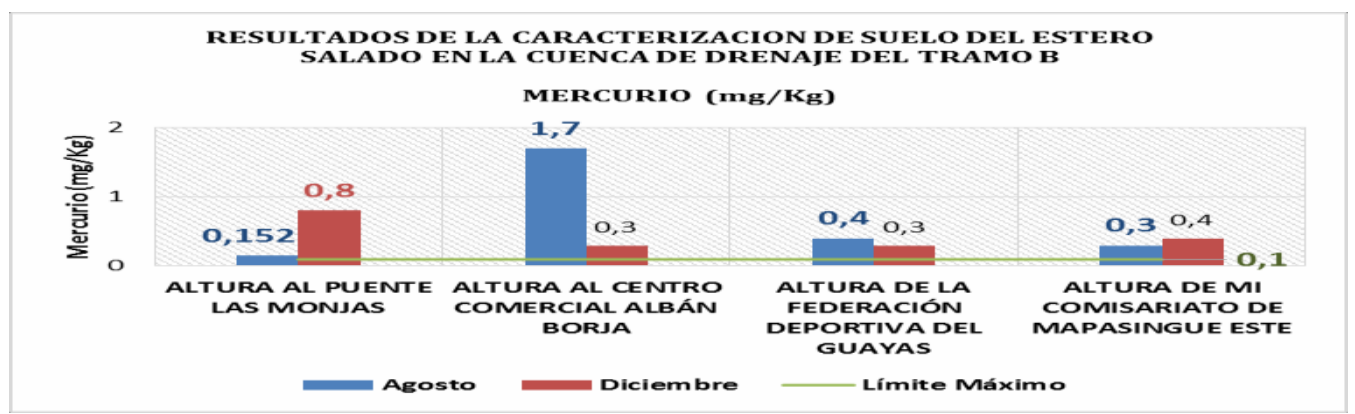

Fig. 23. Resultados de caracterización de Mercurio en el sedimento del Estero Salado

Plomo $(\mathrm{Pb})$

De acuerdo a los resultados obtenidos en la caracterización del sedimento (Fig. 24) se observa que en la estación 1, 2 los meses agosto y diciembre, y en la estación 3 y 4 en el mes de diciembre sobrepasan el límite máximo (19 mg/Kg) de metal, mientras que en agosto en la estación 3 y 4 se encuentran por debajo del límite.

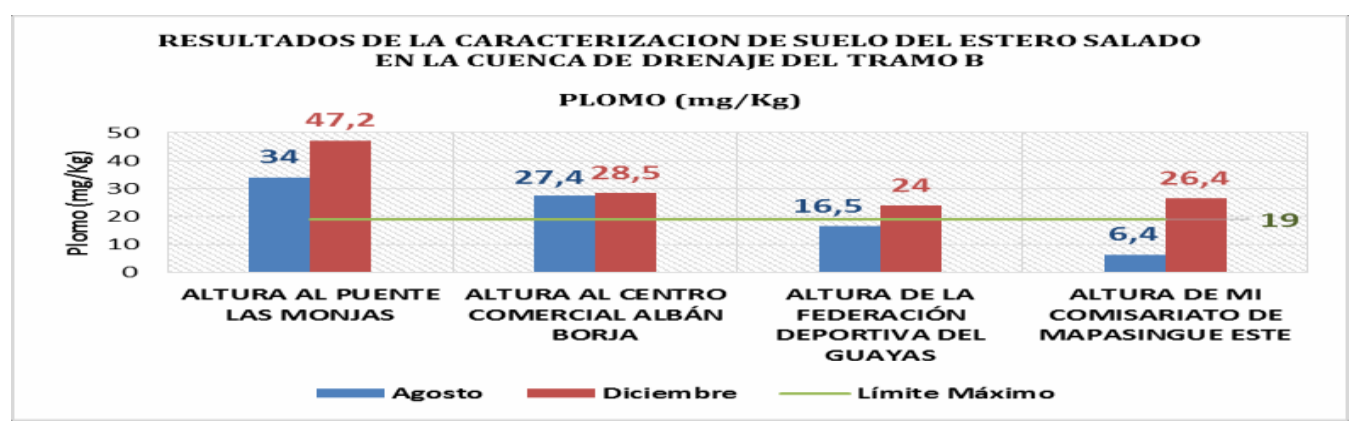

Fig. 24. Resultados de caracterización de Plomo en el sedimento del Estero Salado

Plan de Acción para Remediación del Estero

De acuerdo al análisis de resultados de cada parámetro, se recomienda un plan de acción para minimizar la contaminación del Estero Salado. (CUADRO VI) 


\begin{tabular}{|c|c|c|}
\hline \multicolumn{3}{|c|}{ PLAN DE REMEDIACIÓN DEL ESTERO SALADO } \\
\hline Paráı & Generación & Plan de Mitigación \\
\hline Oxígeno Disuelto & \multirow{4}{*}{$\begin{array}{l}\text { Descargas de aguas } \\
\text { residuales domésticas } \\
\text { e industriales sin } \\
\text { previo tratamiento o } \\
\text { que no cumple con la } \\
\text { normativa ambiental } \\
\text { vigente. }\end{array}$} & \multirow{4}{*}{$\begin{array}{l}\text { Para elevar la concentración de } \\
\text { oxígeno disuelto se recomienda } \\
\text { implementar un sistema de aireación } \\
\text { mediante instalación de Blower's en } \\
\text { diferentes puntos del estero, así } \\
\text { aumentar los niveles del mismo. Al } \\
\text { mismo tiempo ayuda a la } \\
\text { degradación de la materia orgánica. }\end{array}$} \\
\hline & & \\
\hline de Oxígeno & & \\
\hline $\begin{array}{l}\text { Demanda Química de } \\
\text { Oxígeno }\end{array}$ & & \\
\hline Metales pesados & $\begin{array}{l}\text { Descargas de aguas } \\
\text { residuales industriales } \\
\text { que no cumplen con la } \\
\text { normativa ambiental } \\
\text { vigente. }\end{array}$ & $\begin{array}{l}\text { Se recomienda control de las } \\
\text { descargas de los efluentes, plantas de } \\
\text { tratamientos de aguas residuales en } \\
\text { las industrias, para que estas cumplan } \\
\text { con los límites permisibles exigidos } \\
\text { por la normativa ambiental vigente. } \\
\text { La regulación de las descargas } \\
\text { controla la contaminación tanto en el } \\
\text { agua como el sedimento en el estero. }\end{array}$ \\
\hline Coliformes Fecales & $\begin{array}{l}\text { Descargas de aguas } \\
\text { residuales domésticas } \\
\text { e industriales sin } \\
\text { previo tratamiento. }\end{array}$ & $\begin{array}{l}\text { Construcción de una planta de } \\
\text { tratamiento de aguas residuales } \\
\text { domésticas en la Ciudad de } \\
\text { Guayaquil. } \\
\text { Control de las descargas de aguas } \\
\text { domésticas en entidades como } \\
\text { Urbanizaciones e Industrias. }\end{array}$ \\
\hline
\end{tabular}

Cuadro 2. Plan de remediación de parámetros físicos-químicos del estero salado 


\section{Conclusiones}

Se concluye que la calidad de agua del Estero Salado de acuerdo a los parámetros analizados, en época de invierno tiene menor concentración de contaminantes en comparación con las condiciones observadas en época de verano de acuerdo a los resultados de las muestras analizadas, debido a que existe una solubilidad de la concentración de los mismo por las entradas de aguas.

Según el monitoreo realizado en las dos estaciones climáticas y horarios de maras se concluye que existe una variación de los valores, es decir, en pleamar (marea alta) los parámetros se encuentran en menor concentración, mientras que en bajamar (marea baja) los valores de los parámetros en el sistema están elevados, lo que indica en el estrato de fondo del estero no hay recambio suficiente, lo cual se puede verificar con los datos obtenidos en la medición de las corrientes, pues los valores de tanto superficiales como de fondo son bajos, por lo que existe una lenta renovación de agua.

De acuerdo a la comparación de los resultados obtenidos de la caracterización de las muestras del Estero Salado se concluye que los parámetros en su mayoría se encuentran fuera de los límites máximos estimados por la normativa ambiental vigente, por lo cual es recomendable aplicar un sistema de gestión para la remediación del mismo.

\section{Agradecimiento}

Los autores agradecen a los laboratorios de la Universidad de Guayaquil y al Laboratorio Químicos Marcos por la ayuda de descuento obtenido por los análisis realizados. 
Evaluación de la calidad físico-químico en agua y sedimentos del estero salado en el noroeste de América Latina

\section{Bibliografía.}

Reche, I. (2003). Sensibilidad de los ecosistemas acuáticos a la radiación ultravioleta Alicante, España: REDALYC.

Acuerdo Ministerial 097-A (2016). Acuerdo Ministerial 097-A. Quito: Ministerio de Medio Ambiente.

Boothroyd, J., H. Ayón, D. Robadue, J. Vásconez, y R. Noboa. 1994. Características de la línea costera del Ecuador y recomendaciones para su manejo. Programa de Manejo de Recursos Costeros. Ecuador.67p.

CAAM 1996. Desarrollo y Problemática Ambiental del Área del Golfo de Guayaquil. 354 p.

Cruz M. 1992. Estado actual del recurso malacológico (Bivalvos y Gasterópodos) de la zona infralitoral del Golfo de Guayaquil. Acta Oceanográfica del Pacífico 7(1): 41-68.

Holland, A.F., Sanger, D.M., Gawle, C.P., Lerberg, S.B., Santiago., M.S., Riekerk, G.H.M., Zimmerman, L.E., Scott, G.E., 2004. Linkages between Tidal Creek Ecosystems and the Landscape and Demographic Attributes of their Waterheds Journal Experimental Marine Biology Vol. 298: 151-178.

Pérez, J. 2012. Caracterización del componente de Flora del Estero Salado en los ramales con diferentes tipos de desarrollo urbano. Informe técnico preparado para Ecobiotec del Ecuador y Ministerio del Ambiente.276p.

Stevenson, M. 1981. Variaciones Estacionales en el Golfo de Guayaquil, un estuario tropical. Boletín Científico y técnico Guayaquil: Instituto Nacional de Pesca.133p.

Valverde, F. D. y J. Pérez. 2012. La biodiversidad vegetal como capital natural de la sostenibilidad en la costa ecuatoriana, Guayaquil, M: I: Municipalidad de Santiago de Guayaquil.797 